\title{
Olaparib for Chinese Metastatic Castration-resistant Prostate Cancer: A Real-World Study of Efficacy and Gene Predictive Analysis
}

\section{Baijun Dong}

Shanghai Jiao Tong University School of Medicine Affiliated Renji Hospital

Bin Yang

Tongji University Tenth People's Hospital: Shanghai Tenth People's Hospital

Wei Chen

Wenzhou Medical College First Affiliated Hospital: The First Affiliated Hospital of Wenzhou Medical University

Xinxing Du

Shanghai Jiao Tong University School of Medicine Affiliated Renji Hospital

Liancheng Fan

Shanghai Jiao Tong University School of Medicine Affiliated Renji Hospital

Xudong Yao

Tongji University Tenth People's Hospital: Shanghai Tenth People's Hospital

Wei Xue ( $\nabla$ xuewei@renji.com )

Shanghai Jiao Tong University School of Medicine Affiliated Renji Hospital https://orcid.org/0000-0003-0124-9433

\section{Research Article}

Keywords: Olaparib, HRR mutations, mCRPC, VUS, BRCA2, PSA, PSA-PFS

Posted Date: November 30th, 2021

DOI: https://doi.org/10.21203/rs.3.rs-1055524/v1

License: @ (1) This work is licensed under a Creative Commons Attribution 4.0 International License. Read Full License 


\section{Abstract}

Objective: To evaluate the real-world effectiveness and gene predictive analysis of olaparib in Chinese patients with metastatic castration-resistant prostate cancer (mCRPC) .

Methods: A multicenter, retrospective, real-world study was conducted by involving Chinese patients with mCRPC from December 2017 to June 2021. Homologous Recombination repair (HRR)gene mutation (HRRm) status was identified using targeted next-generation sequencing (NGS). The primary endpoint includes prostate-specific antigen (PSA) response rate (PSA $\left.A_{50}\right)$. Secondary end points include PSA progression-free survival (PSA-PFS), exploratory endpoints include PSA ${ }_{50}$, and PSA-PFS in HRRm-negative patients with variants of unknown significance (VUS). Survival rates were analyzed using Kaplan-Meier (KM) plot.

Results: A total of 39 eligible patients with a median age of 65 (interquartile range [IQR]: 59.5-69.5) years were included in the study. Overall, $40 \%$ (12/30) of the patients with mCRPC achieved PSA 50 and the median PSA-PFS was 3.1 months (95\% Confidence interval [Cl]: 2.4-7). Furthermore, higher $\mathrm{PSA}_{50}$ rate and longer PSA-PFS were observed in HRRm-positive patients (PSA 50 : 50\% [7/14]; median PSA-PFS: 5.3 months, 95\% Cl: 3.73-10). Among the HRRm-positive patients, those harboring the BRCA2 aberrations experienced best clinical efficacy (PSA ${ }_{50}: 55.5 \%$ [5/9] and median PSA-PFS [95\% Cl]: 9.5 months [4.3, NA]). Clinical benefit was also observed in HRRmnegative patients (PSA50: 31.3\% [5/16]; median PSA-PFS [95\% Cl]:2.05 months [1.5,8]), wherein most patients with a PSA 50 response were carrying VUS mutations (PSA 50 : 50\% [4/8]; median PSA-PFS [95\% Cl]: 2.75 months [1.27, NA]). In one patients with mutation in the ATR gene, the PSA level decreased by $62 \%$.

Conclusion: Olaparib improved PSA response and prolonged PSA-PFS in Chinese mCRPC patients especially in those carrying HRR mutation. Among the HRR genes, patients with BRCA2 mutation showed the best clinical benefit. Besides, some patients carrying HRR VUS alternations and other DNA damage response (DDR) gene mutations also showed response to olaparib treatment, indicating that the clinical benefits observed in HRR negative group were driven by VUS and other DDR gene mutation. However, this should be further explored in the future, and more molecular functional studies are needed to reclassify VUS mutations for better clinical treatment decision-making and management of mCRPC.

\section{Introduction}

Metastatic castration-resistant prostate cancer ( $\mathrm{MCRPC}$ ) is a heterogeneous disease generally associated with aberrations in the DNA damage response (DDR) genes leading to poor clinical outcomes. ${ }^{1}$ However, the therapeutic landscape of mCRPC has rapidly evolved with the advancement in sequencing techniques such as targeted next-generation sequencing (NGS) for the identification of mutations that could guide the application of targeted therapy. NGS also revealed that mCRPC tumors has a complex genomic landscape wherein genomic alterations were observed in pathways that are not related to androgen receptors and which could be targeted for therapy. ${ }^{2,3}$ Meanwhile, several studies from China demonstrated that there are a large number of patients with prostate cancer carrying mutations in homologous recombination repair (HRR) genes. ${ }^{4,5}$ In addition, $15 \%$ to $30 \%$ of cases were found with germline or somatic mutations in HRR genes (including BRCA1/2, ATM and CHEK2) and have more aggressive disease and higher mortality than those with proficient HRR functionality. ${ }^{6-9}$ Therefore, the high prevalence of these mutations has led to the National Comprehensive Cancer Network (NCCN) recommending germline/somatic testing of HRR gene alterations in all patients with metastatic prostate cancer. ${ }^{10}$

Recently, studies have demonstrated that the presence of such genetic aberrations may enhance the sensitivity of prostate cancer to poly (adenosine diphosphate-ribose) polymerase (PARP) inhibitors.$^{11}$ The PROfound trial which evaluated the efficacy of olaparib in prostate cancer patients with HRR gene aberrations demonstrated significant increase in progression free survival in olaparib treated patients in comparison to standard of care. ${ }^{1}$ Based on the results of the PROfound trial, the NCCN guideline recommended treatment with olaparib for patients with HRR-mutated mCRPC. ${ }^{10}$ Subsequently, in May 2020, the Food and Drug Administration approved olaparib in adult patients with mCRPC with germline/somatic HRR gene alterations. ${ }^{12}$ Furthermore, the National Medical Products Administration (NMPA) approved olaparib in MCRPC patients carrying germline and/or somatic BRCA1/2 mutations who have progressed after prior treatment with enzalutamide or abiraterone in June 2021. Likewise, several studies demonstrated the differential clinical outcomes with olaparib in patients with HRR gene mutated mCRPC such as BRCA1/2 orATM. ${ }^{13,14}$ Despite these remarkable observations, the current clinical decisions are guided mainly by evidence from western populations. Besides, genomic study in Chinese patients with metastatic prostate cancers suggests that germline mutation spectrum of HRR genes is similar among Chinese and Western population. ${ }^{4}$ However, there are some differences in the somatic mutation spectrums where the prevalence of BRCA2 (13\%) and CDK12 (15.4\%) mutations in 
tumor tissue is higher in Chinese mCRPC population, and patients with CDK12 defects showed rapid disease progression after abiraterone treatment while patients with BRCA2 defects showed marked response to PARP inhibitors and platinum based chemotherapy. ${ }^{15,16}$ Furthermore, there still lack of real-world evidence from China evaluating the effectiveness of olaparib in patients with mCRPC. Therefore, this real-world study was undertaken to evaluate the effectiveness of spontaneous use of olaparib in Chinese patients with $\mathrm{MCRPC}$.

In addition, there are no hotspot mutations in HRR genes and the classification of the pathogenicity of HRR mutations are based on the statistics in research reports and the degree to which the genetic change is predicted to alter the structure and function of the encoded protein, and classified as pathogenic, likely pathogenic, variants of uncertain significance(VUS), likely benign and benign. ${ }^{17}$ HRR mutations which have not been reported previously or conflict evidence existed on the functionality effect of encoded protein are classified as VUS, and clinical decision cannot be made if patients carrying VUS mutation. However, the proportion of the VUS in HRR genes is high due to the lack of evidence, where $30 \%$ of genetic tests reported VUS alterations, ${ }^{18}$ which leads to confusion in clinical practice, and needs to be further explored desperately.

\section{Methods}

\section{Study Design and Data Collection}

A multicenter, retrospective, real-world study was conducted by including Chinese patients with mCRPC. Patients' demographic and clinical data were obtained from Renji Hospital affiliated to Shanghai Jiaotong University School of Medicine, the Tenth People's Hospital affiliated to Shanghai Tongji University, and the First affiliated hospital of Wenzhou Medical University, China, from December 2017 to June 2021. The HRR gene mutation status (BRCA1/2, ATM, PALB2, CDK12, RAD54L, RAD51B,,RAD51C,RAD51D, FANCL, CHEK1/2, BRIP1, and BARD1) of patients with mCRPC were confirmed by targeted NGS using deep targeted sequencing of plasma circulating tumor DNA (ctDNA) or tumor tissue. Furthermore, patients who received previous olaparib (at least 1 month) mono or combo therapy spontaneously and no prior treatment with other PARP inhibitors were included in the study. In addition, data for systemic therapy initiated after the onset of MCRPC were obtained. For each systemic therapy, the data on treatment line and the decline in PSA $\geq 50 \%$ from baseline (PSA ${ }_{50}$ ) were obtained. All centers participating in the study obtained approval from the local institutional review board before data abstraction.

\section{Next-Generation Sequencing and Bioinformatic Analysis}

The targeted NGS test of all tissue/blood samples collected from each individual patient was performed at Glorious Med Clinical Laboratory (Shanghai) Co, Ltd. Targeted sequencing and bioinformatic analysis, including the identification of germline and somatic mutation and copy number alteration, were conducted as described previously by Dong et al. ${ }^{15}$ The pathogenicity of identified gene variants was interpreted by following the general guideline of germline mutation and somatic mutation classification. ${ }^{19,20}$ All loss-offunction alterations of HRR genes were coded as deleterious or suspected deleterious, including deletion, nonsense mutations, frameshift, and splice site alterations. Otherwise, unless specifically designated as deleterious in the ClinVar database, missense mutations were considered as VUS. Patients carrying deleterious/suspected deleterious mutation on HRR gene (BRCA1/2, ATM, PALB2, CDK12, RAD54L, RAD51B/C/D, FANCL, CHEK1/2, BRIP1, and BARD1) were considered as the HRRm-positive group and others were considered as the HRRm-negative group, which includes patients carrying VUS on HRR gene (HRRm-negative VUS) or those carrying other DDR defects or no deleterious mutations identified (HRR-negative other).

\section{Treatment and Outcomes}

Patients who received systemic therapy with olaparib either as monotherapy or in combination with abiraterone spontaneously were considered and analyzed individually. The primary end point includes PSA response rate (defined as post-medicine PSA decline $>50 \%$ from baseline at 12 weeks). The secondary end points include PSA progression-free survival (PSA-PFS), the exploratory endpoints included PSA response rate, and PSA-PFS in HRR-negative VUS patients. PSA-PFS was defined as the time from the beginning of treatment with olaparib to the first confirmation of PSA progression (defined as testosterone meets the castration level $[<50 \mathrm{ng} / \mathrm{dL} \mathrm{h}$ or $<1.7 \mathrm{nmol} / \mathrm{L}$ ] and blood PSA rises) or death (any cause), whichever occurs first. 


\section{Statistical Analysis}

Continuous variables were represented using values of interquartile range (IQR). Categorical variables were represented in percentages. The reduction in PSA levels $>50 \%$ from the baseline at 12 weeks was considered for PSA remission and $95 \%$ confidence interval (Cl) was calculated using Binomial "exact" calculation. Survival rates were calculated using the Kaplan-Meier (KM) plots. The difference in hazards function was analyzed using the Cox proportional hazard regression analysis, and the results were expressed as hazard ratio $(\mathrm{HR})$ and $95 \% \mathrm{Cl}$. All the analyses were performed with R software, version 3.5. The sorting intolerant from tolerant (SIFT) algorithm was used for predicting the deleterious effect of coding variants on protein function.

\section{Results}

\section{Patient Characteristics and Genomic Landscape}

A total of 39 eligible patients aged 65 (IQR: 59.5-69.5) years with were included in the study. Their data on demographics, clinicopathological characteristics, and prior treatments were presented in Table 1. At the time of diagnosis, the PSA level (IQR) was 110 $(51.5-110) \mathrm{ng} / \mathrm{mL}$, and $81.8 \%$ patients were primarily diagnosed with bone metastasis. Furthermore, $87.2 \%(n=34)$ of the patients have received prior new hormonal agent therapy and 61.5\% $(n=24)$ of the patients have received docetaxel treatment. Besides, olaparib was mainly used in the second line and later phases of treatment predominantly as monotherapy. Moreover, gene test by NGS revealed HRRm-positive results in 18 patients, and the most commonly detected HRR aberrations were in $\operatorname{BRCA2}(\mathrm{n}=10), \operatorname{ATM}(\mathrm{n}=3)$, and $C D K 12$

$(\mathrm{n}=2)$. In addition, HRRm-negative VUS alterations were detected in 8 patients, and the most common VUS mutations detected were in $\operatorname{BRCA2}(\mathrm{n}=3)$ and $\operatorname{ATM}(\mathrm{n}=3)$.

Table 1

\section{Clinicopathological Characteristics}




\begin{tabular}{|c|c|c|c|c|}
\hline & \multirow[t]{2}{*}{ Overall } & \multirow[t]{2}{*}{ HRR Positive } & \multicolumn{2}{|l|}{ HRR Negative } \\
\hline & & & $\begin{array}{l}\text { HRR-Negative } \\
\text { VUS }\end{array}$ & $\begin{array}{l}\text { HRR-Negative } \\
\text { Other }\end{array}$ \\
\hline Sample size (n) & 39 & 18 & 8 & 13 \\
\hline Age (IQR), years & $65(59.5-69.5)$ & $64(59.5-66)$ & $66(59-69.75)$ & $66(59.25-71.5)$ \\
\hline$\geq 65$ years, $n(\%)$ & $19(51.4 \%)$ & $7(18.9 \%)$ & $4(50 \%)$ & $8(61.5 \%)$ \\
\hline PSA (IQR), ng/mL & $\begin{array}{l}110(51.5- \\
110)\end{array}$ & $\begin{array}{l}105(52.8- \\
110)\end{array}$ & $79(34.5-852.5)$ & $\begin{array}{l}110(78.05- \\
257.15)\end{array}$ \\
\hline \multicolumn{5}{|l|}{ Gleason score } \\
\hline$<8$ & $6(16.2 \%)$ & $2(11.8 \%)$ & $1(14.3 \%)$ & $3(23.1 \%)$ \\
\hline$\geq 8$ & $31(83.8 \%)$ & $15(88.2 \%)$ & $6(85.7 \%)$ & $10(76.9 \%)$ \\
\hline Metastasis at primary diagnosis, $\mathrm{n}(\%)$ & $29(87.9 \%)$ & $16(94.1 \%)$ & $4(80 \%)$ & $9(81.8 \%)$ \\
\hline Bone metastasis, n (\%) & $27(81.8 \%)$ & $14(82.4 \%)$ & $4(80 \%)$ & $9(81.8 \%)$ \\
\hline \multicolumn{5}{|l|}{ Previous treatment before Olaparib } \\
\hline $\begin{array}{l}\text { Second-generation androgen receptor inhibitors, } n \\
(\%)\end{array}$ & $34(87.2 \%)$ & $15(83.3 \%)$ & $6(75 \%)$ & $13(100 \%)$ \\
\hline Docetaxel, n (\%) & $24(61.5 \%)$ & $12(66.7 \%)$ & $5(62.5 \%)$ & $7(53.8 \%)$ \\
\hline Platinum-based chemotherapy, n (\%) & $4(10.3 \%)$ & $1(5.6 \%)$ & $1(12.5 \%)$ & $2(15.4 \%)$ \\
\hline \multicolumn{5}{|l|}{ Olaparib treatment } \\
\hline Olaparib monotherapy & $33(84.6 \%)$ & 15 (83.3\%) & $6(85.7 \%)$ & $12(85.7 \%)$ \\
\hline Olaparib + abiraterone & $6(15.4 \%)$ & $3(16.7 \%)$ & $1(12.5 \%)$ & $2(15.4 \%)$ \\
\hline
\end{tabular}

\section{Olaparib Showed Remarkable Clinical Outcomes in Chinese Patients With MCRPC}

$\mathrm{PSA}_{50}$ response and PSA-PFS to olaparib-based treatment were evaluated in all patients. In total, $40 \%(12 / 30)$ of the patients with available data achieved a PSA 50 response, and the response rate is higher in HRRm-positive patients (50\% [7/14]) than in HRRmnegative patients (31.3\% [5/16]; Fig. 1A and B). Meanwhile, $\mathrm{PSA}_{50}$ responders with HRRm-positive alterations majorly harbor $B R C A 2$ mutations $(\mathrm{n}=5)$ followed by CDK12 mutation $(\mathrm{n}=1)$ and RAD51C mutation $(\mathrm{n}=1)$, whereas PSA $\mathrm{A}_{50}$ responders in the HRRm-negative group were mainly carrying HRR VUS mutations in BRCA2 and ATM genes. Overall, the median PSA-PFS in 34 patients with available data was 3.1 months (95\% Cl: 2.4,7). The median PSA-PFS was higher in the HRRm-positive group (5.3 months [95\% Cl: 3.73,10]) compared with the HRRm-negative group (2.05 months [95\% Cl: 1.5-8]; [HR: 0.523; 95\% Cl: 0.265,1.03]; Fig. 2A). Notably, a remarkable clinical benefit was observed in patients with BRCA2 mutation (median PSA-PFS: 9.5 months [95\% Cl: 4.3, NA]; Fig. 2B). The clinical benefits in HRR negative group were mainly observed in patients with HRR VUS mutations and other DDR mutations which elucidated in the following data.

\section{Clinical Benefits of Olaprib Observed in part of Patients With HRR VUS Mutations}

In the present study, there are 8 patients with HRRm-negative VUS mutations and 2 patients with other DDR gene mutations. The mutation sites and effectiveness data of patients with HRR VUS mutations were presented in Table 2. A median PSA-PFS (95\% Cl) of 
2.75 months $(1.27, \mathrm{NA})$ and a PSA 50 response rate of $50 \%$ (4/8) were observed in these patients (Fig. 1C). Particularly, 4 patients with BRCA2 p.L3125P, BRCA2 p.Q1551K, gATM p.Y171C and gATM p.F1036L exhibited longer PSA-PFS (Table 2). Mutation plots showing the placement of the mutation on BRCA2 and ATM are depicted in Figure 3. The mutation on BRCA2 gene was located at the oligonucleotide/oligosaccharide-binding domain 3 and repeat domain (Fig. $3 \mathrm{~A}$ and $\mathrm{B}$ ), whereas, the mutation on ATM was located at the telomere length maintenance-DNA damage repair domain (Fig. 3C). Additionally, bioinformatics prediction algorithm 'sorting intolerant from tolerant' (SIFT) revealed all 3 VUS mutations have damaging impact on the protein structure, indicating its most likely pathogenic nature and clinical relevance, consistent with their better sensitivity to olaparib. In one patient carrying deleterious gene aberration in ATR p.K2106fs, a PSA remission of $62 \%$ was observed.

Table 2

HRR-Negative VUS Mutation Sites and Their Effectiveness Data

\begin{tabular}{|llll|}
\hline Mutation & PSA-PFS (months) & PSA Response Rate (\%) & SIFT Prediction of Protein Function \\
\hline gBRCA2 p.L3125P & 8 & -83.5 & Damage \\
\hline BRCA2 p.Q1551K & 21.4 & NA & Damage \\
\hline gATM p.Y171C & 3.6 & -70.6 & Damage \\
\hline gPALB2 p.P405A & 1.5 & 100 & Tolerated \\
\hline gATM p.Q2802R & 0.5 & 228.6 & Tolerated \\
\hline gCDK12 p.R663C & 1.27 & -61.2 & Not score \\
\hline gBRCA2 p.H2090R & 1.2 & 135 & Tolerated \\
\hline gATM p.F1036L & 4 & -91 & Tolerated \\
\hline $\begin{array}{l}\text { HRR, homologous recombination repair; PSA, prostate-specific antigen; PSA-PFS, prostate-specific antigen progression-free survival; } \\
\text { SIFT, sorting intolerant from tolerant; VUS, variants of uncertain significance. }\end{array}$ \\
\hline
\end{tabular}

\section{Discussion}

Genomic profiling is increasingly used for routine clinical management of prostate cancer. Genomic profiling with NGS has revealed mCRPC to be heterogenous and has also made it feasible for personalized targeted therapy for specific biomarkers. ${ }^{7}$ In the current realworld study, olaparib showed remarkable clinical outcomes in Chinese patients with mCRPC, especially in patients harboring HRR gene aberrations. The results from the current study are in line with the earlier reports from larger prospective trials including the TOPARP-A, TOPARP-B and PROfound trial in which olaparib demonstrated prolonged radiologic progression-free survival (rPFS) in mCRPC patients with HRR mutations. ${ }^{11,21}$ In the PROfound trial, the median rPFS was significantly longer in the patients with at least 1 aberration in HRR genes than in the control group (enzalutamide or abiraterone; rPFS: 5.8 months vs 3.5 months; HR: $0.49 ; 95 \%$ Cl: $0.38-0.63 ; P<0.001$ ). Further $43 \%(66 / 153)$ of patients carrying at least 1 aberration in the BRCA1/2 or ATM gene treated with olaparib had a PSA 50 response while only $8 \%$ of patients in the control group had a $\mathrm{PSA}_{50}$ response. ${ }^{1}$

Several prospective genomic studies demonstrated that men with BRCA1/2 mutations have increased risk of early-onset and clinically significant prostate cancer. ${ }^{22-24}$ Furthermore, germline BRCA1/2 status is an independent prognostic factor for prostate cancer outcome, where patients carrying BRCA1/2 mutation are associated with high Gleason score, late TNM stage at diagnosis, early distant metastasis, and low 5-year survival. ${ }^{25}$ In a prospective cohort study (PROREPAIR-B), cause specific survival of mCRPC patients with BRCA1/2 mutation was significantly less $(17.4 \mathrm{~m}$ vs $33.2 \mathrm{~m}, \mathrm{p}=0.027)$ in comparison to patients with wild type after receiving standard therapy (abiraterone or doxetaxel). ${ }^{26}$ However, in the current study, mCRPC patients harboring BRCA2 mutations experienced superior clinical outcomes with olaparib monotherapy (PSA-PFS: 9.5 months, $95 \% \mathrm{Cl}: 4.3, \mathrm{NA}$ ) which was in accordance to another real-world study wherein patients harboring BRCA 1/2 gene variants had a significantly higher rate of PSA remission and longer PFS than those with variants in other HRR genes. ${ }^{27}$ 
Several studies substantiated that $B R C A 1 / 2$ aberrations are a strong predictor of a favorable response to PARP inhibitors. But in case of prostate cancer, an increase in the frequency of the incidence of VUS in BRCA1/2 gene was reported. ${ }^{28}$ Since the effects of the VUS on the function of the encoded protein or disease risk is unknown, this poses a major challenge for genetic counseling and clinical management, which may require dynamic changes in the reporting of results for clinical decision making ("upgraded" to pathogenic or "downgraded" to benign). ${ }^{29-31}$ However, the findings from the current study demonstrated that patients harboring HRR VUS mutations exhibited a high PSA response rate and prolonged PSA-PFS after olaparib treatment, indicating that patients harboring HRR VUS mutations may benefit from olaparib treatment, which need further confirmation. There are several molecular- or cellular-level functional assays (such as cellular survival and viability assay, DNA recombination repair assay, genomic instability assay, and drug sensitivity assay) with high sensitivity and specificity that allow accurate assessment of the effects of VUS mutations on the encoded protein function. ${ }^{29,30,32}$ Despite considerable efforts to determine the pathogenicity of VUS mutations using multiple functional assays, for most VUS, this is not successful because of the lack of general consensus in clinical practice and evidence on the clinical efficacy of these mutations. ${ }^{31,33}$ To the best of our knowledge, this was the first study to emphasize on the clinical relevance of HRR VUS alterations in Chinese patients with $\mathrm{mCRPC}$; the findings from the current study may bring new evidence to the reclassification of VUS mutations in clinical practice and olaparib maybe considered for mCRPC patients carrying VUS alterations and without effective therapy regimen available.

Another interesting observation in the study is some patients exhibited prolonged survival benefits defined as PFS $>1$ year; the characteristic of these patients is listed in Table 3. Several common points are identified. Four patients carrying BRCA2 mutations (3 patients in the HRRm-positive group and 1 patient with HRR VUS defect) received olaparib and abiraterone combination therapy either as 2L or 3L therapy. In addition, in 1 patient (patient ID: WY) with HRR VUS alteration gATM p.Y171C, PSA level raised after 4 months of treatment and declined after 9 months and demonstrated lower PSA-PFS (4 months). However, the patient exhibited prolonged survival with olaparib treatment (overall survival: 38 months; Table 3). Although PARP inhibitors have demonstrated the clinical effectiveness in several kinds of tumors with underlying HRR deficiencies, there is now biologic and early clinical evidence to support their use in other molecular subsets of cancer, including DDR defects. ${ }^{34,35}$ At this juncture, another noteworthy finding was observed in our study: prolonged PSA-PFS (13 months) and PSA response were observed in a patient harboring DDR defect in the ATR loci (ATR p.K2106fs; Table 3), suggesting that other DDR gene aberrations may also confer sensitivity to olaparib treatment. The role of ATR in DNA repair pathway pertains to both single and double strand break repair. ${ }^{36}$ Mutations in ATR loci is rarely reported in clinical studies and a previous meta-analysis evaluating the role of DDR mutations in 77 genes reported a high number of studies with unknown ATR gene status. ${ }^{37,38}$ Recently, a mounting body of evidence from in vitro and in vivo studies indicates that PARP inhibitors in combination ATR inhibitors can be used across a wide spectrum of cancers, and these findings lead to early-phase clinical trials combining ATR and PARP inhibitors (NCT02576444, NCT02723864, NCT03787680 [TRAP-trial], and NCT03682289). ${ }^{39}$ However, larger prospective trials are required to substantiate the effectiveness of PARP inhibitors in subset of patients with other DDR defects.

Table 3

\section{Patient Characteristics and Clinical Outcomes of Patient Exhibiting Prolonged Survival Benefits}


clinical evidence for the reclassification of these VUS sites, further functional studies and larger prospective trials are needed in this direction to confirm the findings.

\section{Abbreviations}

mCRPC: Metastatic castration-resistant prostate cancer

HRR: Homologous recombination repair

VUS: Variants of uncertain significance

PSA: Prostate-specific antigen

PSA-PFS: Prostate-specific antigen progression-free survival

NGS: Next-generation sequencing

\section{Declarations}

\section{Funding}

This study was supported by the National Natural Science Foundation of China $(81572536,81672920,81772742,81702840,81702542$, 31570993), the Science and Technology Commission of Shanghai Municipality (14140901700, 16411969800, 19411967400), the Shanghai Shenkang Hospital Development Center (SHDC12015125, 16CR3049A), the Shanghai Municipal Education Commission (15ZZ058), the Shanghai Municipal Commission of Health and Family Planning (201640247), the Shanghai Municipal Education Commission-Gaofeng Clinical Medicine Grant Support (20152215), Shanghai Jiao Tong University (YG2016ZD08, YG2017MS47, YG2017MS52); the Innovation Fund for Translational Research of Shanghai Jiao Tong University School of Medicine (15ZH4002), and the Incubating Program for clinical Research and Innovation of Renji Hospital Shanghai Jiao Tong University School of Medicine (PYZY 16-008, PYXJS16-015).

\section{Author Contribution}

Conception and design: Baijun Dong, Wei Chen , Xinxing Du, Liancheng Fan, Xudong Yao, Bing Yang, Wei Xue

Acquisition of data (acquired and managed patients, provided facilities, etc.): Baijun Dong, Liancheng Fan, Bin Yang, Wei Chen and Wei Xue.

Analysis and interpretation of data (e.g., statistical analysis, biostatistics, and computational analysis): Baijun Dong and Liancheng Fan Writing, review, and/or revision of the manuscript: Baijun Dong, Liancheng Fan

\section{Conflict of Interest}

No conflict of interest claimed.

\section{Acknowledgments}

The authors acknowledge the support on data collection, data analysis and medical writing provided by Min Jin, Tingting Zhao and Yanyan Peng, Yihong Liu.

\section{References}

1. de Bono J, Mateo J, Fizazi K, et al. Olaparib for Metastatic Castration-Resistant Prostate Cancer. N Engl J Med. 2020;382(22):20912102. doi:10.1056/NEJMoa1911440 
2. Robinson D, Van Allen EM, Wu Y-M, et al. Integrative clinical genomics of advanced prostate cancer. Cell. 2015;161(5):1215-1228. doi:10.1016/j.cell.2015.05.001

3. van Dessel LF, van Riet J, Smits M, et al. The genomic landscape of metastatic castration-resistant prostate cancers reveals multiple distinct genotypes with potential clinical impact. Nat Commun. 2019;10(1):5251. doi:10.1038/s41467-019-13084-7

4. Wei Y, Wu J, Gu W, et al. Germline DNA Repair Gene Mutation Landscape in Chinese Prostate Cancer Patients. Eur Urol. 2019;76(3):280-283. doi:10.1016/j.eururo.2019.06.004

5. Wu J. Prevalence of comprehensive DNA damage repair gene germline mutations in Chinese prostate cancer patients. International Journal of Cancer. 2020;148(3):673-681. doi:10.1002/ijc.33324

6. Pritchard CC, Mateo J, Walsh MF, et al. Inherited DNA-Repair Gene Mutations in Men with Metastatic Prostate Cancer. New England Journal of Medicine. 2016;375(5):443-453. doi:10.1056/NEJMoa1603144

7. Abida W, Armenia J, Gopalan A, et al. Prospective Genomic Profiling of Prostate Cancer Across Disease States Reveals Germline and Somatic Alterations That May Affect Clinical Decision Making. JCO Precis Oncol. 2017;2017. doi:10.1200/P0.17.00029

8. Jang A, Sartor O, Barata PC, Paller CJ. Therapeutic Potential of PARP Inhibitors in the Treatment of Metastatic Castration-Resistant Prostate Cancer. Cancers (Basel). 2020;12(11). doi:10.3390/cancers12113467

9. Hussain M, Mateo J, Fizazi K, et al. Survival with Olaparib in Metastatic Castration-Resistant Prostate Cancer. N Engl J Med. 2020;383(24):2345-2357. doi:10.1056/NEJMoa2022485

10. NCCN Guidelines for Prostate Cancer V.1.2022. https://www.nccn.org/login?

ReturnURL=https://www.nccn.org/professionals/physician_gls/pdf/prostate.pdf. Accessed October 22, 2021.

11. Mateo J, Porta N, Bianchini D, et al. Olaparib in patients with metastatic castration-resistant prostate cancer with DNA repair gene aberrations (TOPARP-B): a multicentre, open-label, randomised, phase 2 trial. Lancet Oncol. 2020;21(1):162-174. doi:10.1016/S1470-2045(19)30684-9

12. FDA approves olaparib for HRR gene-mutated metastatic castration-resistant prostate cancer. https://www.fda.gov/drugs/drugapprovals-and-databases/fda-approves-olaparib-hrr-gene-mutated-metastatic-castration-resistant-prostate-cancer.

13. Kwon DH, Chou J, Yip SM, et al. Differential treatment outcomes in BRCA1/2-, CDK12-, and ATM-mutated metastatic castrationresistant prostate cancer. Cancer. March 2021. doi:10.1002/cncr.33487

14. Marshall CH, Sokolova AO, McNatty AL, et al. Differential Response to Olaparib Treatment Among Men with Metastatic Castrationresistant Prostate Cancer Harboring BRCA1 or BRCA2 Versus ATM Mutations. Eur Urol. 2019;76(4):452-458. doi:10.1016/j.eururo.2019.02.002

15. Dong B, Fan L, Yang B, et al. Use of Circulating Tumor DNA for the Clinical Management of Metastatic Castration-Resistant Prostate Cancer: A Multicenter, Real-World Study. J Natl Compr Canc Netw. 14 2021:1-10. doi:10.6004/jnccn.2020.7663

16. Fan L, Fei X, Zhu Y, et al. Comparative Analysis of Genomic Alterations across Castration Sensitive and Castration Resistant Prostate Cancer via Circulating Tumor DNA Sequencing. J Urol. 2021;205(2):461-469. doi:10.1097/JU.0000000000001363

17. What's a "Variant of Uncertain Significance?" A VUS? DNA Science. May 2018. https://dnascience.plos.org/2018/05/03/whats-avariant-of-uncertain-significance-a-vus/. Accessed October 22, 2021.

18. Scott RJ, Mehta A, Macedo GS, Borisov PS, Kanesvaran R, El Metnawy W. Genetic testing for homologous recombination repair (HRR) in metastatic castration-resistant prostate cancer (mCRPC): challenges and solutions. Oncotarget. 2021;12(16):1600-1614. doi:10.18632/oncotarget.28015

19. Richards S, Aziz N, Bale S, et al. Standards and guidelines for the interpretation of sequence variants: a joint consensus recommendation of the American College of Medical Genetics and Genomics and the Association for Molecular Pathology. Genet Med. 2015;17(5):405-424. doi:10.1038/gim.2015.30

20. Li MM, Datto M, Duncavage EJ, et al. Standards and Guidelines for the Interpretation and Reporting of Sequence Variants in Cancer: A Joint Consensus Recommendation of the Association for Molecular Pathology, American Society of Clinical Oncology, and College of American Pathologists. J Mol Diagn. 2017;19(1):4-23. doi:10.1016/j.jmoldx.2016.10.002

21. Mateo J, Carreira S, Sandhu S, et al. DNA-Repair Defects and Olaparib in Metastatic Prostate Cancer. N Engl J Med. 2015;373(18):1697-1708. doi:10.1056/NEJMoa1506859

22. Nyberg T, Frost D, Barrowdale D, et al. Prostate Cancer Risks for Male BRCA1 and BRCA2 Mutation Carriers: A Prospective Cohort Study. European Urology. 2020;77(1):24-35. doi:10.1016/j.eururo.2019.08.025 
23. Page EC, Bancroft EK, Brook MN, et al. Interim Results from the IMPACT Study: Evidence for Prostate-specific Antigen Screening in BRCA2 Mutation Carriers. European Urology. 2019;76(6):831-842. doi:10.1016/j.eururo.2019.08.019

24. Kote-Jarai Z, Leongamornlert D, Saunders E, et al. BRCA2 is a moderate penetrance gene contributing to young-onset prostate cancer: implications for genetic testing in prostate cancer patients. Br J Cancer. 2011;105(8):1230-1234. doi:10.1038/bjc.2011.383

25. Castro E, Goh C, Olmos D, et al. Germline BRCA mutations are associated with higher risk of nodal involvement, distant metastasis, and poor survival outcomes in prostate cancer. J Clin Oncol. 2013;31(14):1748-1757. doi:10.1200/JC0.2012.43.1882

26. Castro E, Romero-Laorden N, Del Pozo A, et al. PROREPAIR-B: A Prospective Cohort Study of the Impact of Germline DNA Repair Mutations on the Outcomes of Patients With Metastatic Castration-Resistant Prostate Cancer. J Clin Oncol. 2019;37(6):490-503. doi:10.1200/JC0.18.00358

27. Price MP. Real-world outcomes among prostate cancer patients with BRCA2 gene variants compared to variants in other homologous DNA repair genes. Journal of Clinical Oncology. 2021;39(15_suppl). doi:10.1200/JC0.2021.39.15_suppl.e17033

28. Petrovics G, Price DK, Lou H, et al. Increased frequency of germline BRCA2 mutations associates with prostate cancer metastasis in a racially diverse patient population. Prostate Cancer Prostatic Dis. 2019;22(3):406-410. doi:10.1038/s41391-018-0114-1

29. Lindor NM, Goldgar DE, Tavtigian SV, Plon SE, Couch FJ. BRCA1/2 sequence variants of uncertain significance: a primer for providers to assist in discussions and in medical management. Oncologist. 2013;18(5):518-524. doi:10.1634/theoncologist.20120452

30. Bouwman P, van der Heijden I, van der Gulden H, et al. Functional Categorization of BRCA1 Variants of Uncertain Clinical Significance in Homologous Recombination Repair Complementation Assays. Clin Cancer Res. 2020;26(17):4559-4568. doi:10.1158/1078-0432.CCR-20-0255

31. Hoffman-Andrews $L$. The known unknown: the challenges of genetic variants of uncertain significance in clinical practice. $J$ Law Biosci. 2017;4(3):648-657. doi:10.1093/jlb/lsx038

32. Ikegami M, Kohsaka S, Ueno T, et al. High-throughput functional evaluation of BRCA2 variants of unknown significance. Nat Commun. 2020;11(1):2573. doi:10.1038/s41467-020-16141-8

33. Mesman RLS, Calléja FMGR, Hendriks G, et al. The functional impact of variants of uncertain significance in BRCA2. Genet Med. 2019;21(2):293-302. doi:10.1038/s41436-018-0052-2

34. Mateo J, Boysen G, Barbieri CE, et al. DNA Repair in Prostate Cancer: Biology and Clinical Implications. Eur Urol. 2017;71(3):417-425. doi:10.1016/j.eururo.2016.08.037

35. Pilié PG, Gay CM, Byers LA, O'Connor MJ, Yap TA. PARP Inhibitors: Extending Benefit Beyond BRCA-Mutant Cancers. Clin Cancer Res. 2019;25(13):3759-3771. doi:10.1158/1078-0432.CCR-18-0968

36. Reichert ZR, Daignault S, Teply BA, Devitt ME, Heath El. Targeting resistant prostate cancer with ATR and PARP inhibition (TRAP trial): A phase II study. JCO. 2020;38(6_suppl):TPS254-TPS254. doi:10.1200/JC0.2020.38.6_suppl.TPS254

37. Swift SL, Lang SH, White H, Misso K, Kleijnen J, Quek RG. Effect of DNA damage response mutations on prostate cancer prognosis: a systematic review. Future Oncology. 2019;15(28):3283-3303. doi:10.2217/fon-2019-0298

38. Annala M, Struss WJ, Warner EW, et al. Treatment Outcomes and Tumor Loss of Heterozygosity in Germline DNA Repair-deficient Prostate Cancer. Eur Urol. 2017;72(1):34-42. doi:10.1016/j.eururo.2017.02.023

39. Lloyd RL, Wijnhoven PWG, Ramos-Montoya A, et al. Combined PARP and ATR inhibition potentiates genome instability and cell death in ATM-deficient cancer cells. Oncogene. 2020;39(25):4869-4883. doi:10.1038/s41388-020-1328-y

\section{Figures}


(A)

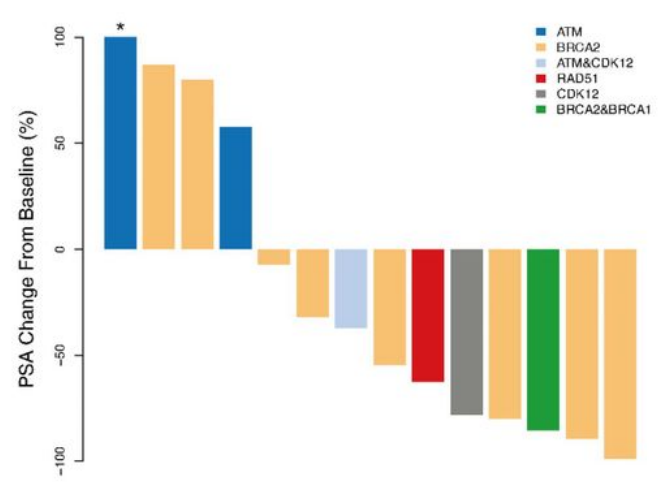

(B) HR negative group

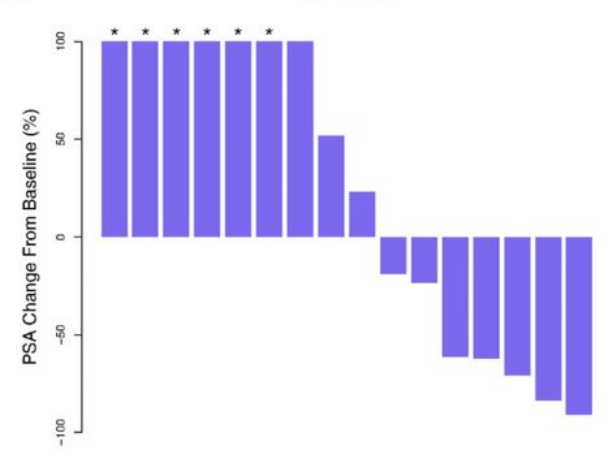

(C) HR negative-VUS group

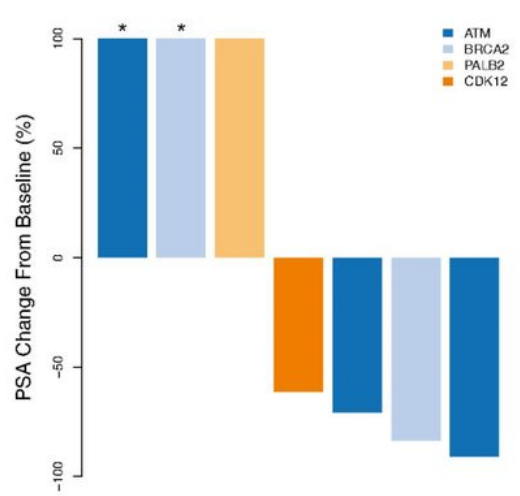

Figure 1. PSA remission rate: (A) HRRm-positive group, (B) HRRm-negative group, and (C) HRRmnegative VUS group. HRR, homologous recombination repair; PSA, prostate-specific antigen ; VUS, variants of uncertain significance; $*$ indicates PSA change $>100 \%$.

\section{Figure 1}

Please See image above for figure legend. 
(A)
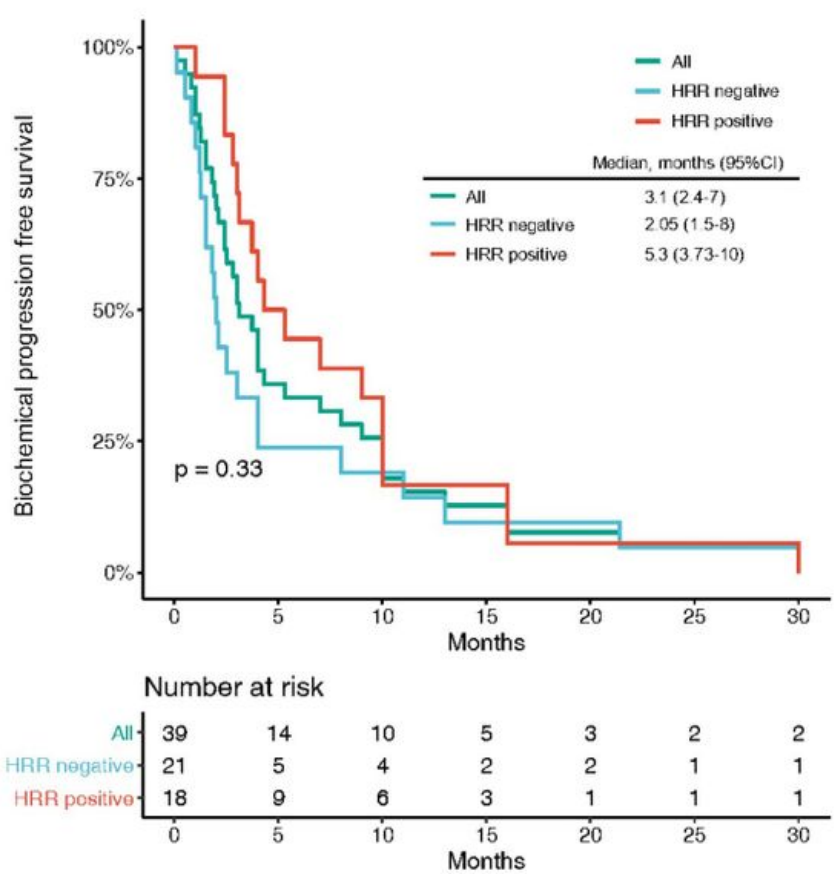

(B)

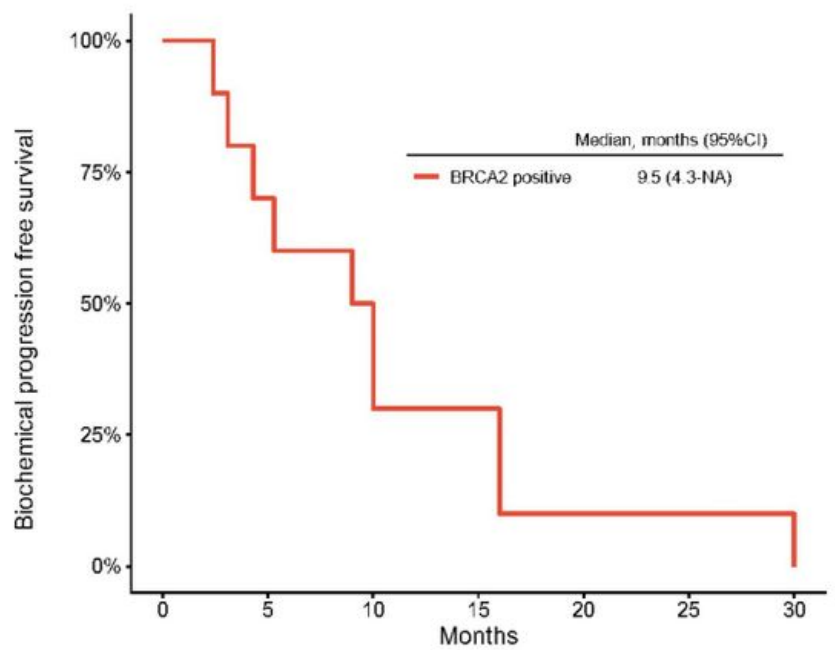

Number at risk

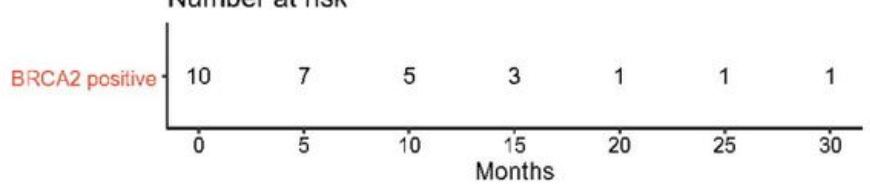

Figure 2. Kaplan-Meier curves for PSA-PFS: (A) overall group, HRRm-positive subgroup, and HRRnegative subgroup and (B) HRRm-positive patients with BRCA2 mutations.

HRR, homologous recombination repair; PSA-PFS, prostate-specific antigen progression free survival

\section{Figure 2}

Please See image above for figure legend. 
(A) BRCA2_p.L3125P.svg

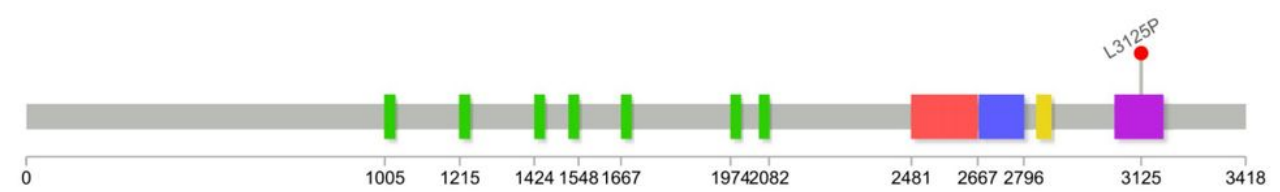

BRCA2, oligonucleotide/oligosaccharide-binding, domain 1

Tower

BRCA2, oligonucleotide/oligosaccharide-binding, domain 3

BRCA2 repeat

BRCA2, helical

(B) BRCA2_p.Q1551K.svg

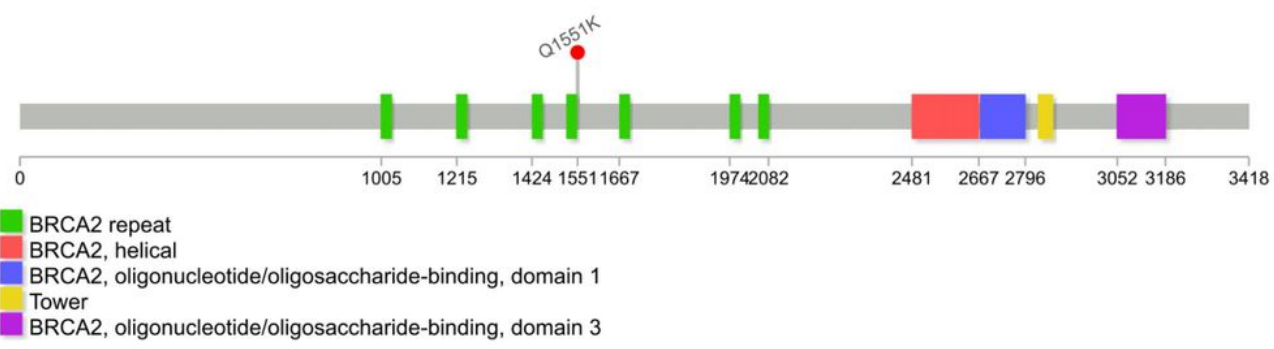

(C) ATM_p.Y171C.svg

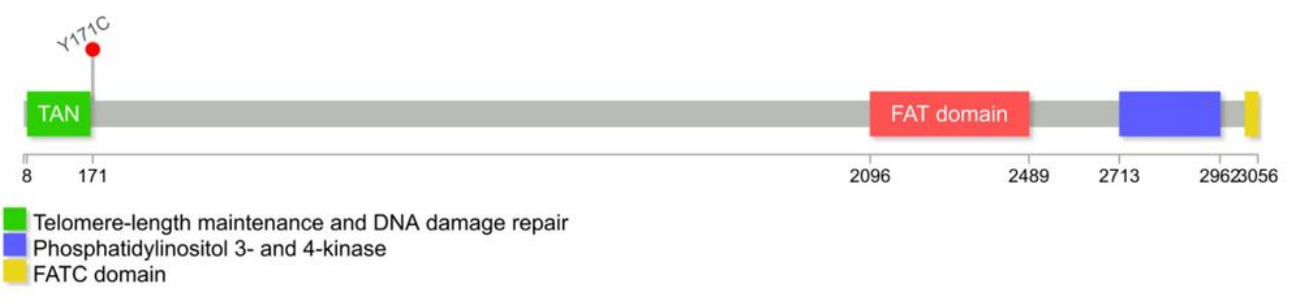

Figure 3. A \&B) Mutations plots in BRCA2 gene

C) Mutation plots in ATM genes. The plots were generated using the online tool Mutation Mapper

\section{Figure 3}

Please See image above for figure legend. 